\title{
Resuscitation practices in cardiac surgery
}

\author{
Igor Gosev, $\mathrm{MD},{ }^{\mathrm{a}}$ Ivana Nikolic, $\mathrm{MD},{ }^{\mathrm{b}}$ and Sari Aranki, $\mathrm{MD}^{\mathrm{a}}$
}

During the past decade, there has been an increasing recognition that cardiac surgery patients have different resuscitative needs than other medical and surgical patients who experience in-hospital cardiac arrest. The special resuscitative needs of cardiac surgery patients were addressed in the 2010 European Resuscitation Council Guidelines for Resuscitation in the section reviewing cardiac arrest in special circumstances and the 2010 American Heart Association (AHA) Guidelines for Cardiopulmonary Resuscitation and Emergency Cardiovascular Care in Special Situations. ${ }^{1,2}$ To date, the most comprehensive and current analysis of survival to hospital discharge after an inhospital cardiac arrest included 84,625 patients at 374 hospitals, $79.3 \%$ of whom had asystole or pulseless electrical activity and $20.7 \%$ of whom had pulseless ventricular tachycardia as their initial rhythm. ${ }^{3}$ Although that study excluded patients in operating rooms, procedural suites, and emergency departments because of their distinct clinical circumstances, the risk-adjusted rate of survival to hospital discharge was $22.3 \%$ in 2009 , markedly improved compared with $13.7 \%$ in 2000 .

Given the continuous monitoring of patients in the intensive care units (ICUs), which decreases the risk of an unwitnessed cardiac arrest and the delay to initiation of cardiopulmonary resuscitation (CPR), cardiac arrests in ICUs represent a subgroup within the in-hospital cardiac arrest group. Although the postcardiac arrest survival rates among the patients in ICUs have varied widely $(0 \%$ $79 \%$ ), cardiac surgery patients seem to fare better. ${ }^{4}$ From 2 separate studies, the survival to discharge among cardiac surgery patients who experienced an in-hospital cardiac arrest was $60 \%$ to $79 \%$, with $45 \%$ to $69 \%$ survival at 1 year follow-up. ${ }^{4-6}$ The differences in survival after a cardiac arrest in the cardiac surgery ICUs compared with other in-hospital cardiac arrests are likely related to the high incidence of cardiac arrests resulting from reversible causes, underscoring the need for, and importance of, a protocolbased, standardized approach to CPR in the cardiac surgery ICU. $^{7}$

From the Division of Cardiac Surgery, ${ }^{\mathrm{a}}$ Department of Surgery, and Cardiovascular Division, ${ }^{\text {b }}$ Department of Medicine, Brigham and Women's Hospital, Harvard Medical School, Boston, Mass.

Disclosures: Authors have nothing to disclose with regard to commercial support.

Received for publication April 6, 2014; revisions received June 10, 2014; accepted for publication June 11, 2014; available ahead of print July 22, 2014.

Address for reprints: Igor Gosev, MD, Division of Cardiac Surgery, Department of Surgery, Brigham and Women's Hospital, Boston, MA (E-mail: igorgosev@ gmail.com).

J Thorac Cardiovasc Surg 2014;148:1152-6

$0022-5223 / \$ 36.00$

Copyright (C) 2014 by The American Association for Thoracic Surgery

http://dx.doi.org/10.1016/j.jtcvs.2014.06.033

\section{EUROPEAN VERSUS AHA GUIDELINES}

The 2010 European Resuscitation Council Guidelines for Resuscitation and the 2010 AHA Guidelines for Cardiopulmonary Resuscitation and Emergency Cardiovascular Care have emphasized the importance of basic life support and uninterrupted, high-quality external cardiac massage $(\mathrm{ECM})$ as a foundation for generating survivable continuous cardiac output. ${ }^{1,2}$ To achieve this, chest compressions in adults should be delivered at a goal rate of $\geq 100$ compressions/min, with a compression depth of $\geq 2$ in, allowing for complete chest recoil with minimal interruptions. Several reports have been published of fatal cardiac complications related to ECM in cardiac surgery patients during the postoperative period. ${ }^{7}$ These reports, coupled with the observation that frequently the cause of cardiac arrest in a cardiac surgery patient can be successfully reversed by defibrillation or pacing, has led to cardiac surgery-specific modifications in the standard postcardiac arrest protocol. The European Guidelines for Resuscitation in Cardiac Surgery Patients created by the Clinical Guidelines Committee of the European Association for CardioThoracic Surgery in 2009 highlight some of the major features that set cardiac surgery patients apart from patients in other ICUs, including several modes of continuous invasive and noninvasive monitoring, fresh, and sometimes very frail, suture lines prone to catastrophic bleeding, and the ability to immediately perform repeat sternotomy. ${ }^{7}$ It is important to note that these guidelines are recommended for use only in cardiac surgery ICUs and that standard advanced cardiac life support (ACLS) protocols should be used in the cardiac surgery step down units.

The most notable modification to the standard ACLS protocol in cardiac surgery ICUs has been the priority given to defibrillation. Because ventricular fibrillation (VF) is the initial rhythm in $25 \%$ to $50 \%$ of cardiac arrests in cardiac surgery ICUs and the recognition of a cardiac arrest is almost immediate owing to the continuous extensive monitoring, in cases of cardiac arrest due to ventricular tachycardia or VF, cardioversion should be attempted before potentially harmful ECM is initiated. Three sequential, stacked attempts of immediate cardioversion without intervening ECM have been recommended for patients with ventricular tachycardia or VF. ${ }^{1,7}$ The success rate for the first, second, and third shock, on average, has been $75 \%, 35 \%$, and $14 \%$, respectively. ${ }^{7}$ If spontaneous rhythm fails to return after the third shock, ECM should be initiated, and the team should proceed to repeat sternotomy. Chan and colleagues ${ }^{8}$ reported that defibrillation that occurred in $<2$ minutes from the arrest carried the best survival and prognosis; therefore, cardioversion should be performed within 

Abbreviations and Acronyms
ACLS $=$ advanced cardiac life support
$\mathrm{AHA}=$ American Heart Association
$\mathrm{CPR}=$ cardiopulmonary resuscitation
$\mathrm{ECM}=$ external cardiac massage
IABP $=$ intra-aortic balloon pump
ICU = intensive care unit
LVAD $=$ left ventricular assist device
$\mathrm{VF}=$ ventricular fibrillation

and the identification of any structures at risk of disruption such as grafts or major suture lines should be performed before 2-handed internal massage is started. According to the European guidelines, chest reentry in cardiac surgery patients is beneficial and safe and should be part of the standard resuscitative protocol until the 10th postoperative day. ${ }^{7}$

\section{RESUSCITATION AFTER MINIMALLY INVASIVE SURGICAL APPROACHES}

Close to 8000 patients have undergone transcatheter aortic valve replacement since its approval by the US Food and Drug Administration for use in inoperable, severe, symptomatic aortic stenosis in 2011 and high-risk, operable aortic stenosis in 2012. ${ }^{9}$ According to The Society of Thoracic Surgeons and American College of Cardiology Transcatheter Valve Therapy Registry, the overall risk of in-hospital cardiac arrest for this patient population in US centers is $5.8 \%$, with an overall in-hospital mortality rate of $5.5 \%$. Several reports have been published of the aortic valve prosthesis deformation found at autopsy after unsuccessful resuscitation efforts in patients who experienced a cardiac arrest after minimally invasive aortic valve replacement. ${ }^{10,11}$

Although generally younger than patients undergoing transcatheter aortic valve replacement and with fewer comorbidities, patients who undergo mini-left thoracotomy and robotic mitral and tricuspid valve interventions have a similar risk of prosthetic valve dehiscence or deformation related to ECM during standard CPR. These specific postoperative situations in which standard ECM could potentially be harmful have not been addressed in the AHA or the European Resuscitation guidelines. However, case reports have suggested that these patients could benefit from a modified resuscitation protocol that would include alternative methods of delivering ECM, the consideration of early median sternotomy and open cardiac massage, and the use of early echocardiography to assess the integrity and function of the valve prosthesis.

\section{CENTRAL ACCESS}

A lack of intravenous access is usually not a problem commonly encountered in a cardiac surgery ICU because cardiac surgery ICU patients usually have multiple including central venous access lines; however, in the spirit of completeness, we should mention intraosseous vascular access as an emerging, ACLS-recommended, rapid, safe, and reliable vascular access option for administration of medications, blood products, and intravenous fluids. The first attempt success rate of intraosseous cannulation has been higher and required less procedure time than the landmark-guided placement of a central venous catheter in a subclavian or femoral vein during a code situation, which has been burdened with high complications and failure rate. ${ }^{12}$ Although intraosseous access does not replace the need for central venous access, it should be considered 


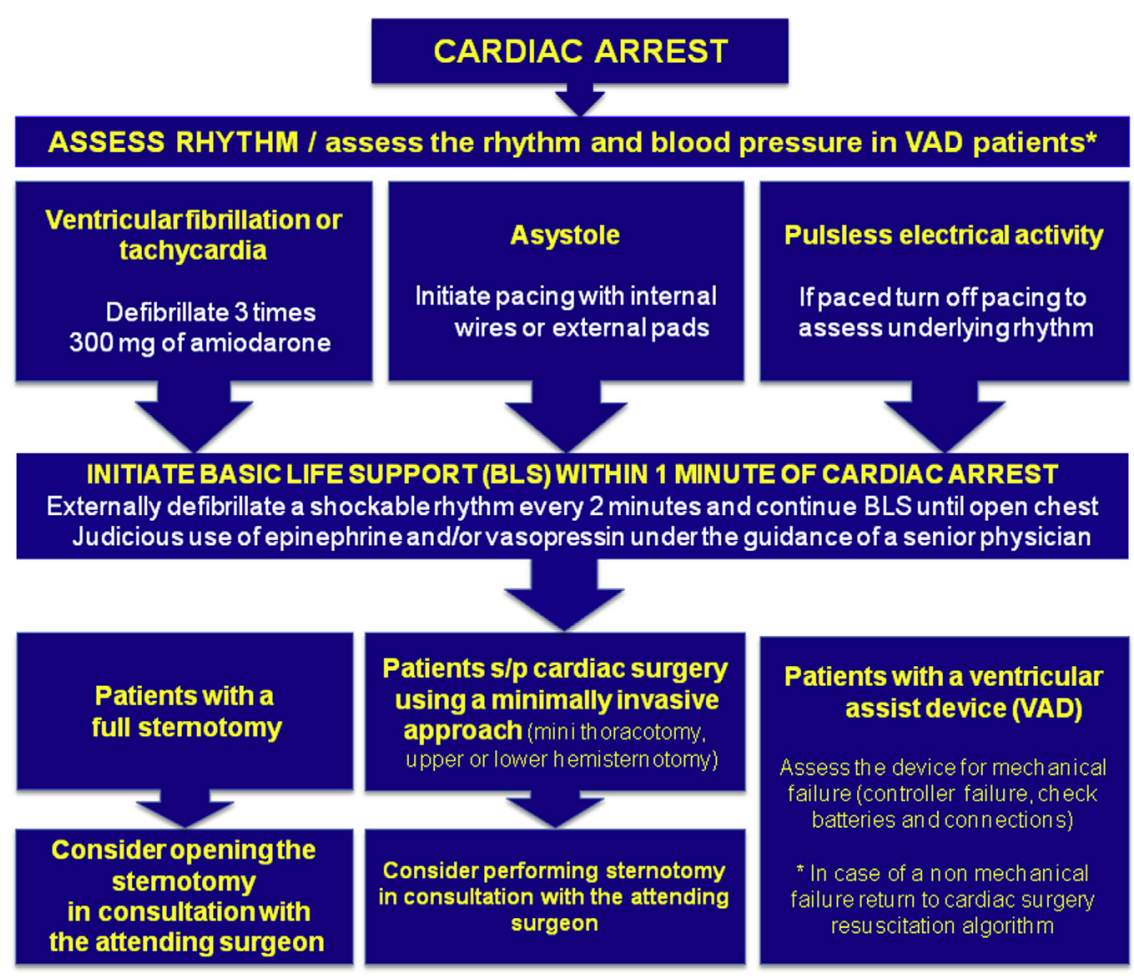

FIGURE 1. Suggested resuscitation protocol for cardiac surgery patients in the cardiac surgery intensive care unit setting. VAD, Ventricular assist device; $s / p$, status post.

as a reliable bridge in a situation in which peripheral or central venous access is not available or cannot be achieved in a timely manner.

\section{MECHANICAL ASSIST DEVICES}

The presence of mechanical assist devices in the cardiac surgery ICU further complicates the management of cardiac arrest in cardiac surgery patients, because cardiac arrest in these patients could be a consequence of mechanical device failure. In such cases, ECM might not be appropriate. Furthermore, the lack of a peripheral pulse in patients with a left ventricular assist device (LVAD) in place makes determination of the occurrence of a cardiac arrest and implementation of the standard ACLS protocol challenging. Although device manufacturers and practitioners have raised concerns regarding potential damage to the inflow or outflow cannula position during standard ECM in LVAD patients, a study of 8 LVAD patients who received standard CPR suggested that standard chest compressions can be safely delivered to LVAD patients. ${ }^{13-15}$ However, the shortest interval from LVAD implantation to the cardiac arrest in that small, retrospective study was 50 days. Thus, the concern that patients with more recent LVAD implantation who receive ECM are at risk of LVAD dislodgement remains.

An animal study has shown abdominal-only CPR to be $60 \%$ more effective than chest-only CPR; however, data to support the use of abdominal-only CPR in patients are lacking. ${ }^{16}$ Studies using interposed abdominal and chest compressions have shown them to be superior to chest compression-only CPR, suggesting that this modified method of CPR might be beneficial, not only in LVAD patients, but also during a period of chest reentry when chest compressions cannot be delivered. ${ }^{16}$ Although patients with mechanical assist devices represent a subgroup of cardiac surgery patients requiring individualized care and staff trained in the specific procedure that pertains to the particular device in use, the approach to patients with an intraaortic balloon pump (IABP) can be more standardized.

The presence of an IABP complicates the resuscitative protocol; however, when operated correctly, the IABP can be used to improve the resuscitative efforts. The presence of an IABP can complicate the recognition of cardiac arrest in the case of pulseless electrical activity arrest or asystole with an active pacemaker. That is, if the IABP has been set to trigger by the electrocardiographic tracing, it will continue to trigger in the aforementioned situations and could produce a pulsatile arterial form, even in the absence of perfusing cardiac output. To avoid such situations, during cardiac arrest, the IABP should be switched to the pressure mode of triggering, with a 1:1 counterpulsation and maximal augmentation, thereby using the IABP for augmentation of the cardiac output generated by the ECM. In cases with a period of no cardiac output, the IABP should be set to the automatic internal mode of 
triggering at a rate of a 100 beats/min. Although suboptimal, this will provide some mechanical circulatory support until meaningful cardiac output has been established by the initiation of ECM, internal cardiac massage, or extracorporeal cardiopulmonary resuscitation (ECPR).

\section{EXTRACORPOREAL CARDIOPULMONARY RESUSCITATION}

No discussion of resuscitation in the modern era cardiac surgery ICUs would be complete without discussing extracorporeal membrane oxygenation and cardiopulmonary bypass. The initiation of ECPR in cardiac surgery patients with a potentially reversible cause of arrest should be considered when available and deemed appropriate. ECPR is an emerging technique that requires a dedicated and well-trained team proficient in peripheral bedside cannulation. Improved survival at discharge and at 1 year of follow-up has been reported for patients started on ECPR after undergoing CPR for $>10$ minutes. ${ }^{17}$ Thiagarajan and colleagues $^{18}$ reported survival to hospital discharge in $27 \%$ of patients from the Extracorporeal Life Support Organization registry, and Haneya and colleagues ${ }^{19}$ reported $34 \%$ survival to hospital discharge for patients receiving extracorporeal membrane oxygenation during CPR. The use of extracorporeal membrane oxygenation or cardiopulmonary bypass in postcardiac surgery patients refractory to standard resuscitation procedures might improve outcomes and is currently a class IIb, level of evidence B recommendation from the AHA Guidelines for Cardiopulmonary Resuscitation.

\section{POSTRESUSCITATIVE CARE}

Until recently, therapeutic hypothermia in the immediate postcardiac arrest period was the only intervention shown to improve neurologic recovery. A multicenter, randomized trial demonstrated an increased rate of favorable neurologic outcomes and reduced 6-month mortality for patients who had experienced an out-of-hospital cardiac arrest and been treated with mild hypothermia $\left(32^{\circ} \mathrm{C}-34^{\circ} \mathrm{C}\right)$ during the immediate 24-hour period after cardiac arrest due to $\mathrm{VF}^{20}$ Despite the recent data that bring to question the benefits of therapeutic hypothermia, the prevailing thought is that active temperature management will remain the standard of care for unconscious survivors of cardiac arrest with successful return of spontaneous circulation. ${ }^{21}$ Although additional studies are needed to delineate the role active temperature management should play during the immediate postcardiac arrest period, therapeutic hypothermia remains a class IIb, level of evidence B recommendation by the 2010 AHA Guidelines for Cardiopulmonary Resuscitation for a comatose, adult patients with a successful return of spontaneous circulation after in-hospital cardiac arrest with any initial rhythm.

\section{CONCLUSIONS}

An immediate postcardiac arrest care algorithm can offer structured guidance to facilitate the identification and treatment of the potential causes of the arrest, prevention of arrest recurrence, optimization of cardiac output and oxygen delivery during the arrest, and implementation of neuroprotective strategies after the return of spontaneous circulation. In the era of minimally invasive cardiac surgery when many cardiac procedures are performed without a full median sternotomy, the existence of standardized protocols for performing chest reentry and the importance of communication between the operating room and ICU staff cannot be overstated. The presence and availability of various mechanical assist devices in the cardiac surgery ICUs further underscores the need for a team approach, communication, and the establishment of policies and algorithms. By highlighting the specific resuscitative needs of cardiac surgery patients, we hope to facilitate the full use of available resources and implementation of standardized protocols (Figure 1), modified to suit the specific clinical scenarios, that will lead to improved survival and an increased likelihood of favorable neurologic outcomes in cardiac surgery ICUs.

\section{References}

1. Soar J, Perkins GD, Abbas G, Alfonzo A, Barelli A, Bierens JJ, et al. European Resuscitation Council Guidelines for Resuscitation 2010 Section 8. Cardiac arrest in special circumstances: electrolyte abnormalities, poisoning, drowning, accidental hypothermia, hyperthermia, asthma, anaphylaxis, cardiac surgery, trauma, pregnancy, electrocution. Resuscitation. 2010;81:1400-33.

2. Vanden Hoek TL, Morrison LJ, Shuster M, Donnino M, Sinz E, Lavonas EJ, et al Part 12: cardiac arrest in special situations: 2010 American Heart Association Guidelines for Cardiopulmonary Resuscitation and Emergency Cardiovascular Care. Circulation. 2010;122:S829-61.

3. Girotra S, Nallamothu BK, Spertus JA, Li Y, Krumholz HM, Chan PS, American Heart Association Get with the Guidelines-Resuscitation Investigators. Trends in survival after in-hospital cardiac arrest. N Engl J Med. 2012;367: 1912-20.

4. Efendijev I, Nurmi J, Castrén M, Skrifvars MB. Incidence and outcome from adult cardiac arrest occurring in the intensive care unit: a systematic review of the literature. Resuscitation. 2014;85:472-9.

5. Guney MR, Ketenci B, Yapici F, Sokullu O, Firat MF, Uvarel H, et al. Results of treatment methods in cardiac arrest following coronary artery bypass grafting. $J$ Card Surg. 2009;24:227-33.

6. Anthi A, Tzelepis GE, Alivizatos P, Michalis A, Palatianos GM, Geroulanos S. Unexpected cardiac arrest after cardiac surgery: incidence, predisposing causes, and outcome of open chest cardiopulmonary resuscitation. Chest. 1998;113: $15-9$.

7. Dunning J, Fabbri A, Kolh PH, Levine A, Lockowandt U, Mackay J, et al. Guideline for resuscitation in cardiac arrest after cardiac surgery. Eur J Cardiothorac Surg. 2009;36:3-28.

8. Chan PS, Krumholz HM, Nichol G, Nallamothu BK, American Heart Association National Registry of Cardiopulmonary Resuscitation Investigators. Delayed time to defibrillation after in-hospital cardiac arrest. N Engl J Med. 2008;358: 9-17.

9. Mack MJ, Brennan J, Brindis R, Carroll J, Edwards F, Grover F, et al. Outcomes following transcatheter aortic valve replacement in the United States. JAMA 2013;310:2069-77.

10. Kirov HM, Bothe W, Breuer W, Clasen I, Mall EG, Schlüter A, et al. Deformation of a transapical aortic valve after cardiopulmonary resuscitation: a potential risk of stainless steel stents. J Am Coll Cardiol. 2012;60:1838.

11. Scherner M, Madershahian N, Strauch JT, Wippermann J, Wahlers T. Transapical valve implantation and resuscitation: risk of valve destruction. Ann Thorac Surg. 2011;92:1909-10. 
12. Leidel BA, Kirchhoff C, Bogner V, Braunstein V, Biberthaler P, Kanz KG. Comparison of intraosseous versus central venous vascular access in adults under resuscitation in the emergency department with inaccessible peripheral veins. Resuscitation. 2012;83:40-5.

13. Rottenberg EM, Heard J, Hamlin R, Sun BC, Awad H. Abdominal only CPR during cardiac arrest for a patient with an LVAD during resternotomy: a case report. $J$ Cardiothorac Surg. 2011;6:91.

14. Pistono M, Corrá U, Gnemmi M, Imparato A, Temporelli PL, Genta FT, et al. How to face emergencies in heart failure patients with ventricular assist device. Int J Cardiol. 2013;168:5143-8.

15. Shinara Z, Bellezzo J, Stahovich M, Cheskes S, Chillcott S, Dembitsky W. Chest compressions may be safe in arresting patients with left ventricular assist devices (LVADs). Resuscitation. 2014;85:702-4.

16. Adam Z, Adam S, Khan P, Dunning J. Could we use abdominal compressions rather than chest compression in patients who arrest after cardiac surgery? Interact Cardiovasc Thorac Surg. 2009;8:148-52.
17. Chen YS, Lin JW, Yu HY, Ko WJ, Jerng JS, Chang WT, et al. Cardiopulmonary resuscitation with assisted extracorporeal life-support versus conventional cardiopulmonary resuscitation in adults with in-hospital cardiac arrest: an observational study and propensity analysis. Lancet. 2008;372:554-61.

18. Thiagarajan RR, Brogan TV, Scheurer MA, Laussen PC, Rycus PT, Bratton SL. Extracorporeal membrane oxygenation to support cardiopulmonary resuscitation in adults. Ann Thorac Surg. 2009;87:778-85.

19. Haneya A, Phillipp A, Diez C, Schopka S, Bein T, Zimmermann M, et al. A 5-year experience with cardiopulmonary resuscitation using extracorporeal life support in non-postcardiotomy patients with cardiac arrest. Resuscitation. 2012;83:1331-7.

20. The Hypothermia after Cardiac Arrest Study Group. Mild therapeutic hypothermia to improve the neurologic outcome after cardiac arrest. N Engl J Med. 2002; 346:549-56.

21. Nielsen N, Wettersley J, Cronberg T, Erlinge D, Gasche Y, Hassager C, et al. Targeted temperature management at $33^{\circ} \mathrm{C}$ versus $36^{\circ} \mathrm{C}$ after cardiac arrest. $N$ Engl J Med. 2013;369:2197-206.

\title{
EDITORIAL COMMENTARY
}

\section{Unique aspects of resuscitation practices in postoperative cardiac surgical care: A call to action}

\author{
Juan Nicolas Pulido, MD
}

Cardiac arrest after cardiac surgery is a relatively infrequent but serious event with an incidence ranging from $0.7 \%$ to $2.9 \%$. ${ }^{1}$ Although this can have potential devastating consequences, reported survival to hospital discharge is usually higher in these patients than in those who have in-hospital cardiac arrest occurring in other settings. ${ }^{1-3}$ This is generally attributed to the immediate recognition capabilities in the intensive care unit, high rate of reversible causes, and rapid access to pacing, defibrillation, mechanical circulatory support, or potentially lifesaving emergency resternotomy. Nevertheless, there is wide variation and lack of standard in resuscitation practices in this patient population, with outcomes largely dependent on the expertise of the personnel in charge at the moment of the event.

From the Division of Cardiac Surgery, ${ }^{\mathrm{a}}$ Department of Surgery, and Cardiovascular Division, ${ }^{\text {b }}$ Department of Medicine, Brigham and Women's Hospital, Harvard Medical School, Boston, Mass.

Disclosures: Author has nothing to disclose with regard to commercial support.

Received for publication Aug 28, 2014; accepted for publication Aug 28, 2014; available ahead of print July 22, 2014

Address for reprints: Juan Nicolas Pulido, MD, Department of Anesthesiology, Mayo Clinic, 200 1st St SW, Mary Brigh Building, Rochester, MN 55905 (E-mail: Pulido.juan@mayo.edu).

J Thorac Cardiovasc Surg 2014;148:1156-7

$0022-5223 / \$ 36.00$

Copyright (c) 2014 by The American Association for Thoracic Surgery

http://dx.doi.org/10.1016/j.jtcvs.2014.08.038
On the contrary, well-established protocols in the general population have streamlined the care of out-of-hospital and in-hospital cardiac arrest. Protocols, such as the well-known Advanced Cardiac Life Support (ACLS) and Basic Life Support (BLS), allow for smooth participation of the resuscitation team with known roles, responsibilities, and expectations in these stressful, otherwise chaotic situations.

For those who routinely care for cardiac surgical patients, it is not surprising to find that this patient population possesses unique characteristics and aspects of care, in whom traditional resuscitation practices can result in harm. Despite the description of this concept in multiple studies $^{1-3}$ and the development of a cardiac surgery life support training course, ${ }^{4}$ to date, there is no universally accepted standardized protocol-based approach to resuscitation in cardiac surgical postoperative care.

The expert review by Gosev and colleagues, ${ }^{5}$ published in this month's issue of the Journal, is a timely and thought-provoking article that describes the unique needs and characteristics of the cardiac surgical patient who experiences a postoperative cardiac arrest and how standard resuscitation techniques guided by ACLS may be detrimental in the acute postoperative setting, unveiling an important gap in contemporary cardiac surgical intensive care. Only recently, the value of specific cardiac surgical protocol-guided resuscitation has been emphasized in detail with a clear goal-directed protocol. ${ }^{1,4}$ The authors review 